\title{
A necessary condition for well-posed Cauchy problems
}

By

\author{
Katsuju IGARI
}

(Received Oct. 24, 1975)

\section{§ 0. Introduction}

Consider the partial differential equation

$$
L[u]=\left(\frac{\partial}{\partial t}\right)^{m} u+\sum_{|v|+j \leq m, j<m} a_{v j}(x, t)\left(\frac{\partial}{\partial x}\right)^{v}\left(\frac{\partial}{\partial t}\right)^{j} u=0
$$

in $\Omega=\boldsymbol{R}^{\prime \prime} \times[0, T], T>0$. Consider the Cauchy problem for this equation with given initial data at $t=0$.

Definition. We say that the Cauchy problem for $(0.1)$ is wellposed in $\mathscr{D}_{L^{2}}^{\infty}$, if for any given initial data $\varphi_{j}(x) \in \mathscr{D}_{L^{2}}^{\infty}, j=0,1, \ldots$, $m-1$, there exists a unique solution $u(x, t) \in \mathscr{E}_{t}^{m}\left(\mathscr{D}_{L^{2}}^{\infty}\right), 0 \leq t \leq T$, which takes the given initial data at $t=0$ :

$$
\left.\left(\frac{\partial}{\partial t}\right)^{j} u(x, t)\right|_{t=0}=\varphi_{j}(x), \quad j=0,1, \ldots, m-1 .
$$

It is an interesting problem to look for a necessary condition in order that the Cauchy problem be well-posed, and this problem was studied by many authors. The following theorem proved by S. Mizohata is one of the most important results.

We consider the characteristic equation

$$
p(\lambda)=\lambda^{\prime \prime \prime}+\sum_{|v|+j=m} a_{v j}(x, t) \xi^{v} \lambda^{j}=0, \quad \xi \in \boldsymbol{R}^{\prime \prime} .
$$


We denote the roots by $\lambda_{j}(x, t ; \xi), j=1,2, \ldots, m$. If the Cauchy problem for (0.1) with initial time $t=0$ is well-posed in $\mathscr{D}_{L^{2}}^{\infty}$, then for any $x \in \boldsymbol{R}^{n}, \xi \in \boldsymbol{R}^{n}$, every root $\lambda_{j}(x, t ; \xi)$ must be real-valued at $t=0$ :

$$
\operatorname{Im} \lambda_{j}(x, 0 ; \xi) \equiv 0, \quad x \in \boldsymbol{R}^{n}, \xi \in \boldsymbol{R}^{n}, j=1,2, \ldots, m .
$$

It is natural to pose a question of whether every root $\lambda_{j}(x, t ; \xi)$ must be real-valued not only at the initial time $t=0$ but also at an arbitrary time $\mathrm{t} \in[0, T]$.

In this paper we treat only the equations whose coefficients depend only on $t$. Namely we consider

$$
L[u]=\left(-\frac{\partial}{\partial t}\right)^{m} u+\sum_{|v|+j \leq m, j<m} a_{v j}(t)\left(\frac{\partial}{\partial x}\right)^{v}\left(\frac{\partial}{\partial t}\right)^{j} u=0
$$

in $\Omega=\boldsymbol{R}^{n} \times[0, T], T>0$. We assume that $a_{v j}(t) \in C^{m-1}[0, T]$ in the case of $|v|+j=m$ and that $a_{v j}(t) \in C^{0}[0, T]$ in the other case. Consider the charateristic equation

$$
p(\lambda)=\lambda^{m}+\sum_{|v|+j=m} a_{v j}(t) \xi^{v} \lambda^{j}=0, \quad \xi \in \boldsymbol{R}^{n} .
$$

We denote the roots by $\lambda_{j}(t ; \xi), j=1,2, \ldots, m$. We define

$$
t_{0}(\xi)=\sup \left\{t ; \operatorname{Im} \lambda_{j}(s ; \xi) \equiv 0,0 \leq s \leq t, j=1,2, \ldots, m\right\},
$$

where we regard $\xi$ as a parameter. Our aim is to prove the

Theorem. If for some $\xi^{0}(\neq 0) \in \boldsymbol{R}^{n}$ the following two conditions are both satisfied, then the Cauchy problem for $\left(0.1^{\prime}\right)$ with initial time $t=0$ is not well-posed in $\mathscr{D}_{L^{2}}^{\infty}$.

Condition $A: \quad t_{0}\left(\xi^{0}\right)<T$.

Condition $B$ : There exists $t_{1} ; t_{0}=t_{0}\left(\xi^{0}\right)<t_{1}<T$, such that every characteristic root $\lambda_{j}\left(t ; \xi^{0}\right)$ belongs to $C^{0}\left[t_{0}, t_{1}\right] \cap C^{m-1}\left(t_{0}, t_{1}\right]$ and satisfies

$$
\left.\operatorname{Im} \lambda_{j}\left(t ; \xi^{0}\right) \equiv 0 \quad \text { or } \quad \operatorname{Im} \lambda_{j}\left(t ; \xi^{0}\right) \neq 0, \quad t_{0}<t \leq t_{1} \cdot{ }^{*}\right)
$$

*) Taking the definition of $t_{0}\left(\tilde{\xi}^{0}\right)$ into account, we may suppose that at least one of roots satisfies the latter. 
Example. Consider the differential equation

$$
\frac{\partial^{2} u}{\partial t^{2}}-\alpha t^{p} \frac{\partial^{2} u}{\partial x^{2}}+(\text { lower order terms })=0,
$$

Where $\alpha$ is a constant, $p$ is a real non-negative constant. It follows from our theorem that the constant $\alpha$ must be real non-negative for the Cauchy problem to be well-posed.

Remark 1. Consider the differential equation

$$
\begin{aligned}
\frac{\partial^{2} u}{\partial t^{2}} & +2 \sum_{j=1}^{n} a_{j}(t) \frac{\partial^{2} u}{\partial x_{j} \partial t}+\sum_{j, k=1}^{n} a_{j k}(t) \frac{\partial^{2} u}{\partial x_{j} \partial x_{k}} \\
& +(\text { lower order terms })=0,
\end{aligned}
$$

in $\Omega=\boldsymbol{R}^{n} \times[0, T]$. Here we assume that $a_{j}(t), a_{j k}(t)$ are all realvalued analytic functions. In this case we can see easily that the condition $B$ is always satisfied. Therefore as a consequence of the theorem we can say that for the Cauchy problem to be well-posed $\lambda_{j}(t ; \xi)$ must be real-valued:

$$
\operatorname{Im} \lambda_{j}(t ; \xi) \equiv 0, \quad 0 \leq t \leq T, \quad \xi \in \boldsymbol{R}^{n}, \quad j=1,2
$$

Remark 2. We can replace the condition $B$ by the following weaker one.

Condition $B^{\prime}$ : There exists $t_{1} ; t_{0}=t_{0}\left(\xi^{0}\right)<t_{1}<T$, such that every characteristic root $\lambda_{j}\left(t ; \xi^{0}\right)$ belongs to $C^{0}\left[t_{0}, t_{1}\right] \cap C^{m-1}\left(t_{0}, t_{1}\right]$. Moreover we can choose $\left\{j_{1}, j_{2}, \ldots, j_{p}\right\}, p \geq 1$, from $\{1,2, \ldots, m\}$ so that

$(* *) \quad\left\{\begin{array}{l}\operatorname{Im} \lambda_{j_{k}}\left(t ; \xi^{0}\right)>0, \quad t_{0}<t \leq t_{1}, \quad k=1,2, \ldots, p, \\ \operatorname{Im} \lambda_{j}\left(t ; \xi^{0}\right)<\min _{k=1, \ldots, p} \operatorname{Im} \lambda_{j_{k}}\left(t ; \xi^{0}\right), \quad t_{0}<t \leq t_{1}, \quad j \notin\left\{j_{1}, \ldots, j_{p}\right\} .\end{array}\right.$

Now we state our plan of the proof. By the Fourier transform of $\left(0.1^{\prime}\right)$ with respect to the space variables we get

$$
\hat{L}[v]=\left(\frac{\partial}{\partial t}\right)^{m} v+\sum_{|v|+j \leq m} a_{v j}(t)(i \xi)^{v}\left(\frac{\partial}{\partial t}\right)^{j} v=0
$$


This is an ordinary differential equation with a parameter $\xi \in \boldsymbol{R}^{n}$. To each solution $v(t ; \xi)$ of $(0.4)$ we consider its energy:

$$
E(t)=\left.\left.\sum_{j=1}^{m}|| \xi\right|^{m-j}\left(\frac{\partial}{\partial t}\right)^{j-1} v(t ; \xi)\right|^{2}
$$

We know the following proposition, [4], cf. also [3].

Proposition. 1 (Petrowski). In order that the Cauchy problem for $\left(0.1^{\prime}\right)$ be well-posed in $\mathscr{D}_{L^{2}}^{\infty}$, it is necessary and sufficient that there exist positive constants $C$ and $p$, independent of $\xi$, such that any solution of $(0.4)$ satisfies

$$
E(t) \leq C(1+|\xi|)^{p} E(0), \quad 0 \leq t \leq T .
$$

Under the assumption of the theorem, we shall construct a sequence of solutions of $(0.4)$ which violates the estimate $(0.6)$ whatever the constants $C$ and $p$ may be.

We should remark that if we assume the smoothness of characteristic roots in $\left[0, t_{0}\left(\xi^{0}\right)\right]$, the proposition 2 in $\$ 2$ can be obtained much more easily. ${ }^{*}$

\section{§1. A preparatory consideration}

In this section we consider the behavior of the roots of algebraic equations perturbed by a small parameter. The following lemma is very simple but useful.

Lemma. We assume that the algebraic equation

*) We shall explain briefly the notations used in this paper: $x=\left(x_{1}, x_{2}, \ldots, x_{n}\right)$. $\xi=\left(\xi_{1}, \xi_{3}, \ldots, \xi_{n}\right) . \nu=\left(\nu_{1}, \nu_{2}, \ldots, \nu_{n}\right), \nu_{j}$ are non-negative integers. $|\nu|=\sum_{j=1}^{n}\left|\nu_{j}\right| \cdot\left(\frac{\partial}{\partial x}\right)^{\nu}$ $=\left(\frac{\partial}{\partial x_{1}}\right)^{\nu_{1}}\left(\frac{\partial}{\partial x_{2}}\right)^{\nu_{2}} \cdots\left(\frac{\partial}{\partial x_{n}}\right)^{\nu_{n}} . u(x) \in \mathscr{D}_{L^{2}}^{\infty}$ means that $u(x)$ and all its derivatives $\left(\frac{\partial}{\partial x}\right)^{y} u(x)$ (in distribution sense) belong to $L^{2}\left(R^{n}\right)$. $\mathscr{D}_{L^{2}}^{\infty}$ provided with the semi-norm $p_{m}(u)=\sum_{|\nu| \sum_{m}}\left\|\left(\frac{\partial}{\partial x}\right)^{\nu} u(x)\right\| \quad$ is a Frechét space. $u(x, t) \in \mathscr{E}_{t}^{m}\left(\mathscr{D}_{L^{z}}^{\infty}\right)$ means that $t \longrightarrow u(x, t) \in \mathscr{D}_{L^{2}}^{\infty}$ is continuously differentiable up to order m. $\varphi(t)$ $\in C^{k}\left[0, t_{0}\right]$ means that $\varphi(t), 0 \leq t \leq t_{0}$, is continuously differentiable up to order $k$. 


$$
f(\lambda)=\lambda^{m}+a_{1} \lambda^{m-1}+\cdots+a_{m}=0
$$

has only real roots. Consider the equation perturbed by a complex number $z$ :

$$
f(\lambda)+z=\lambda^{m}+a_{1} \lambda^{m-1}+\cdots+\left(a_{m}+z\right)=0 .
$$

The discriminant $D(z)$ of this equation is a polynomial of $z$ of degree $m-1$ and has only real roots.

Proof. From its definition,

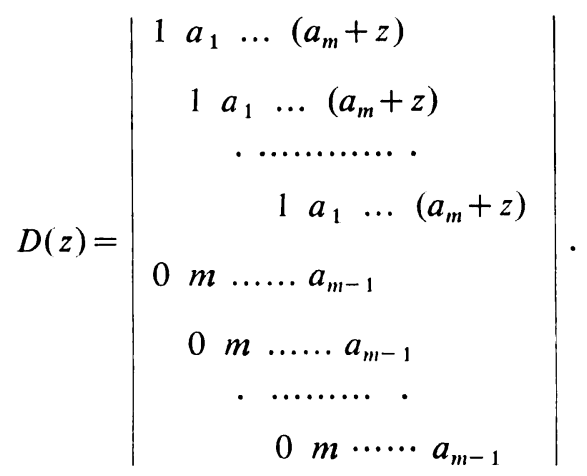

This is a polynomial of $z$ of degree $m-1$ of the form:

$$
D(z)=m^{m} z^{m-1}+b_{1} z^{m-2}+\cdots+b_{m-1} .
$$

$D(z)=0$ means that the two equations:

$$
f(\lambda)+z=0, \quad f^{\prime}(\lambda)=0
$$

have at least one common root. If $\operatorname{Im} z \neq 0$, the first equation has no real root. On the other hand the second one has only real roots, because (1.1) has only real roots. Therefore $D(z)$ has only real roots. q.e.d.

Now we consider the equation of $\lambda$

$$
f(\lambda ; t)=\lambda^{m}+a_{1}(t) \lambda^{m-1}+\cdots+a_{m}(t)=0,
$$


$0 \leq t \leq t_{0}$, where $a_{j}(t) \in C^{m-1}\left[0, t_{0}\right], j=1,2, \ldots, m$. we assume that for any $t \in\left[0, t_{0}\right]$, this equation has only real roots. It is a matter of course that the roots of $\left(1.1^{\prime}\right)$ are not necessarily differentiable with respect to $t$.

Consider the equation perturbed by $z$ :

$$
f(\lambda ; t)+z=\lambda^{m}+a_{1}(t) \lambda^{m-1}+\cdots+\left\{a_{m}(t)+z\right\}=0 .
$$

By the above lemma, we see that the discriminant $D(z ; t)$ of this equation is a polynomial of $z$ of degree $m-1$ and has only real roots. Therefore we may express $D(z ; t)$ as follows:

$$
D(z ; t)=m^{m} \prod_{j=1}^{m-1}\left\{z-\beta_{j}(t)\right\}
$$

where $\beta_{j}(t)$ are real-valued.

Now we put $z=i \varepsilon, \varepsilon>0$. Then it follows from the above expression that

$$
|D(i \varepsilon ; t)| \geq m^{m} \varepsilon^{m-1} .
$$

This means that for any $t \in\left[0, t_{0}\right]$, the equation

$$
\lambda^{m}+a_{1}(t) \lambda^{m-1}+\cdots+a_{m}(t)+i \varepsilon=0
$$

has distinct roots. We denote them by $v_{j}(t ; \varepsilon), j=1,2, \ldots, m . \quad v_{j}(t ; \varepsilon)$ belongs to $C^{m-1}\left[0, t_{0}\right]$.

We put

$$
m(\varepsilon)=\min _{j \neq k} \inf _{0 \leq t \leq t_{0}}\left|v_{j}(t ; \varepsilon)-v_{k}(t ; \varepsilon)\right| .
$$

Then we have the following estimate:

$$
m(\varepsilon) \geq c_{0} \varepsilon^{\frac{m-1}{2}}, \quad c_{0} \text { be a positive constant. }
$$

Here we used the well-known relation

$$
D(i \varepsilon ; t)=(-1)^{\frac{m-1}{2}} \prod_{j<k}\left\{v_{j}(t ; \varepsilon)-v_{k}(t ; \varepsilon)\right\}^{2}
$$


Using (1.6), we obtain the following estimates:

$$
\left|v_{j}^{(k)}(t ; \varepsilon)\right| \leq C \varepsilon^{-\frac{k}{2}(m-1)^{2}-\frac{k-1}{2}(m-1)}, \quad 0 \leq t \leq t_{0},
$$

where $v_{j}^{(k)}(t ; \varepsilon)$ stands for $\frac{d^{k}}{d t^{k}} v_{j}(t ; \varepsilon), C$ is a positive constant, $j=1,2, \ldots$, $m, k=1,2, \ldots, m-1$.

Proof of (1.7). For simplicity, we show this only in case of $k=1$.

$$
f(\lambda ; t)+i \varepsilon=\prod_{j=1}^{m}\left\{\lambda-v_{j}(t ; \varepsilon)\right\}
$$

Differentiating with respect to $t$,

$$
\left.\frac{\partial}{\partial t} f(\lambda ; t)\right|_{\lambda=v_{j}(t ; \varepsilon)}=-v_{j}^{\prime}(t ; \varepsilon) \prod_{k \neq j}\left\{v_{j}(t ; \varepsilon)-v_{k}(t ; \varepsilon)\right\}
$$

Therefore by (1.6)

$$
\left|v_{j}^{\prime}(t ; \varepsilon)\right| \leq C \varepsilon^{-\frac{(m-1)^{2}}{2}} .
$$

The following inequalities are also useful in the next section.

$$
\left|\operatorname{Im} v_{j}(t ; \varepsilon)\right| \leq 2 \varepsilon^{\frac{1}{m}}, \quad 0 \leq t \leq t_{0}, \quad j=1,2, \ldots, m .
$$

By Rouché's theorem, we can see this easily.

\section{§ 2. Energy estimate in $\left[0, t_{0}\left(\xi^{0}\right)\right]$}

In the equation (0.4) we take $\xi=\tau \xi^{\circ}, \tau>0$. Namely we consider the ordinary differential equation with a parameter $\tau$

$$
\hat{L}[v]=\left(\frac{\partial}{\partial t}\right)^{m} v+\sum_{|v|+j \leq m} a_{v j}(t)\left(i \tau \xi^{0}\right)^{v}\left(\frac{\partial}{\partial t}\right)^{j} v=0 .
$$

We define the operator $\hat{L}_{0}$ by

$$
\hat{L}_{0}=\left(\frac{\partial}{\partial t}\right)^{m}+a_{1}(t)(i \tau)\left(\frac{\partial}{\partial t}\right)^{m-1}+\cdots+a_{m}(t)(i \tau)^{m}+i(i \tau)^{m} \tau^{-\sigma}
$$

where $a_{j}(t)=\sum_{|v|=j} a_{v, m-j}(t)\left(\xi^{0}\right)^{v}, j=1,2, \ldots, m, \quad \sigma \quad$ is a positive constant 
which shall be determined later.

By the definition of $t_{0}=t_{0}\left(\xi^{0}\right)$, the characteristic equation

$$
\lambda^{m}+a_{1}(t) \lambda^{m-1}+\cdots+a_{m}(t)=0
$$

has only real roots always in $\left[0, t_{0}\right]$. So we can use the results prepared in the preceding section, taking $\varepsilon=\tau^{-\sigma}$. We denote by $v_{j}(t ; \tau)$, $j=1,2, \ldots, m$, the roots of the equation

$$
\lambda^{m}+a_{1}(t) \lambda^{m-1}+\cdots+a_{m}(t)+i \tau^{-\sigma}=0, \quad 0 \leq t \leq t_{0} .
$$

We define the operators $\partial_{j}$ and $\pi_{j}$ by

$$
\partial_{0}=1, \quad \partial_{j}=\frac{\partial}{\partial t}-i v_{j}(t ; \tau) \tau, \quad j=1,2, \ldots, m,
$$

$$
\pi_{j}=\partial_{j} \partial_{j-1} \cdots \partial_{0}, \quad j=0,1, \ldots, m .
$$

Taking the estimate (1.7) into account, we put $\sigma=\frac{2}{m(m-1)}$. Then $\pi_{m}-\hat{L}_{0}$ can be represented as follows:

$$
\pi_{m}-\hat{L}_{0}=\sum_{k=1}^{m-1}\left\{b_{k 1}(t ; \tau) \tau^{k}+\cdots+b_{k k}(t ; \tau) \tau\left(\frac{\partial}{\partial t}\right)^{k-1}\right\} \pi_{m-k-1}
$$

where $\left.\left|b_{k j}(t ; \tau)\right| \leq C \tau^{1-\frac{1}{m}}, j=1,2, \ldots, k, k=1,2, \ldots, m-1 . *\right)$

Next we express $\left(\frac{\partial}{\partial t}\right)^{j}$ in terms of $\partial_{k}$, i.e.

$$
\begin{aligned}
\left(\frac{\partial}{\partial t}\right)^{j}= & \partial_{k+j-1} \cdots \partial_{k}+c_{1}(t ; \tau) \tau \partial_{k+j-2} \cdots \partial_{k}+\cdots \\
& +c_{j-1}(t ; \tau) \tau^{j-1} \partial_{k}+c_{j}(t ; \tau) \tau^{j}
\end{aligned}
$$

where $\left|c_{l}(t ; \tau)\right| \leq$ constant. Here we used (1.7) again.

By (2.5) and (2.6) we have

$$
\pi_{m}-\hat{L}=c_{1}(t) \pi_{m-1}+c_{2}(t ; \tau) \tau^{1+p} \pi_{m-2}+\cdots+c_{m}(t ; \tau) \tau^{m-1+p} \pi_{0},
$$

*) Hereafter we may use the the symbols $C, M, \ldots$ in order to represent positive constants which can be chosen independently of $\tau$. 
where $\quad p=1-\min \left\{\frac{1}{m}, \frac{2}{m(m-1)}\right\}, \quad\left|c_{1}(t)\right| \leq$ constant, $\quad\left|c_{j}(t ; \tau)\right| \leq$ constant, $j=2,3, \ldots, m$.

By the relations

$$
\tau^{m-j} \pi_{j-1} v=\tau^{-j \alpha} v_{j}, \quad j=1,2, \ldots, m, \alpha=\frac{1-p}{m},
$$

we get the system of equations equivalent to (2.1):

$$
\frac{\partial}{\partial t} \vec{v}=A \tau \vec{v}+B \tau^{1-\alpha} \vec{v}, \quad 0 \leq t \leq t_{0}
$$

where $\vec{v}={ }^{t}\left(v_{1}, v_{2}, \ldots, v_{m}\right)$,

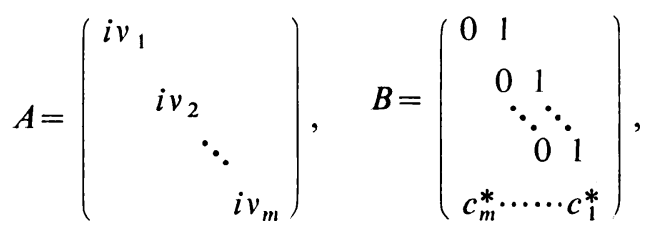

$\left|c_{j}^{*}(t ; \tau)\right| \leq$ constant $, j=1,2, \ldots, m$.

Now we put

$$
S(t)=\sum_{j=1}^{m}\left|v_{j}(t ; \tau)\right|^{2} .
$$

We remark that by (1.8)

$$
\left|\operatorname{Re}\left(i v_{j}\right)(t ; \tau)\right| \leq 2 \tau^{-\frac{2}{m^{2}(m-1)}}
$$

Then

$$
\begin{aligned}
\frac{d}{d t} S(t) & =2 \sum_{j=1}^{m} \operatorname{Re}\left\{v_{j} \frac{\overline{\partial v_{j}}}{\partial t}\right\} \\
& \geq-\left\{C_{1} \tau^{1-\rho}+C_{2} \tau^{1-\alpha}\right\} S(t), \quad \rho=\frac{2}{m^{2}(m-1)} .
\end{aligned}
$$

Because $\alpha=\frac{1-p}{m}=\min \left\{\frac{1}{m^{2}}, \frac{2}{m^{2}(m-1)}\right\} \leq \rho$,

$$
\frac{d}{d t} S(t) \geq-C \tau^{1-\alpha} S(t)
$$


Integrating this inequality, we have

$$
S(0) \leq \exp \left\{C \tau^{1-\alpha}\right\} S\left(t_{0}\right) .
$$

Corresponding to $(0.5)$, we put

$$
E(t)=\sum_{j=1}^{m}\left|\tau^{m-j}\left(\frac{\partial}{\partial t}\right)^{j-1} v(t)\right|^{2} .
$$

$E(t)$ and $S(t)$ are equivalent in the following sense:

$$
m E(t) \leq S(t) \leq M \tau^{2} E(t), \quad 0 \leq t \leq t_{0},
$$

where $m$ and $M$ are positive constants independent of $\tau$. Here we used (2.6). Thus we have the

Proposition 2. For any solution $v(t ; \tau)$ of $(2.1)$ the following energy estimate holds:

$$
E(0) \leq \exp \left\{M_{1} \tau^{1-\alpha}\right\} E\left(t_{0}\right), \quad \text { for large } \tau,
$$

where $\alpha=\min \left\{\frac{1}{m^{2}}, \frac{2}{m^{2}(m-1)}\right\}, M_{1}$ is a positive constant.

\section{§3. Proof of the theorem*)}

Consider the equation (2.1) in $\left[t_{0}, t_{1}\right]$. Without any loss of generality we may assume that for some $k \geq 1$

$$
\begin{aligned}
& -\operatorname{Im} \lambda_{j}(t)>0, \quad t_{0}<t \leq t_{1}, \quad j=1,2, \ldots, k, \\
& -\operatorname{Im} \lambda_{j}(t) \leq 0, \quad t_{0}<t \leq t_{1}, \quad j=k+1, \ldots, m,
\end{aligned}
$$

where $\lambda_{j}(t)=\lambda_{j}\left(t ; \xi^{0}\right)$ are roots of the characteristic equation $\left(0.2^{\prime}\right)$, which belong to $C^{0}\left[t_{0}, t_{1}\right] \cap C^{m-1}\left(t_{0}, t_{1}\right]$.

We define the operators $\tilde{\partial}_{j}$ and $\tilde{\pi}_{j}$ by

$$
\tilde{\partial}_{0}=1, \quad \tilde{\partial}_{j}=\frac{\partial}{\partial t}-i \lambda_{j}(t) \tau, \quad j=1,2, \ldots, m,
$$

*) The argument in this section is due to the idea of S. Mizohata, cf. [2]. 


$$
\tilde{\pi}_{j}=\partial_{j} \partial_{j-1} \ldots \partial_{0}, \quad j=0,1, \ldots, m \text {. }
$$

Then $\tilde{\pi}_{m}-\hat{L}$ can be expressed as follows:

$$
\tilde{\pi}_{m}-\hat{L}=\sum_{k=0}^{m-1} p_{k}(\tau ; t) \tilde{\pi}_{m-k-1}, \quad t_{0}<t \leq t_{1},
$$

where $p_{k}(\tau ; t)=\sum_{j=0}^{k} c_{k j}(t) \tau^{k-j}$. We should remark that $c_{k j}(t)$ are continuous in $\left(t_{0}, t_{1}\right]$ but not necessarily bounded. However if we put $M(t)=\left\{1+\max _{\substack{j=1, \ldots, m \\ k=1, \ldots, \ldots, m-1}}\left|\lambda_{j}^{(k)}(t)\right|\right\}^{m-1}$, then

$$
\left|c_{k j}(t)\right| \leq C M(t), \quad t_{0}<t \leq t_{1},
$$

where $C$ is a positive constant.

By the relations

$$
\tau^{m-j} \tilde{\pi}_{j-1} v=\tau^{-j \alpha} v_{j}, \quad j=1,2, \ldots, m, \quad \alpha=\frac{1}{m},
$$

we get the system of equations equivalent to (2.1):

$$
\frac{\partial}{\partial t} \vec{v}=A \tau \vec{v}+B \tau^{1-\alpha} \vec{v}
$$

where $\vec{v}={ }^{t}\left(v_{1}, v_{2}, \ldots, v_{m}\right)$,

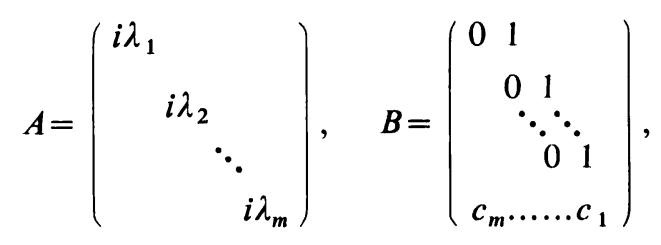

$\left|c_{j}(\tau ; t)\right|=\left|\tau^{-(j-1)-1+j \alpha} p_{j-1}(\tau ; t)\right| \leq C M(t)$.

Now we set $2 \delta(t)=\min _{j=1, \ldots, k}\left\{-\operatorname{Im} \lambda_{j}(t)\right\}$. Because of $(3.1), \delta(t)>0$ for $t \in\left(t_{0}, t_{1}\right]$. We define $v_{j}^{*}(t)$ by

$$
v_{j}^{*}(t)=v_{j}(t) \exp \left\{-\tau \int_{t_{0}}^{t} \delta(s) \mathrm{d} s\right\} .
$$

Then we obtain 


$$
\frac{\partial}{\partial t} \vec{v}^{*}=A^{*} \tau \vec{v}^{*}+B \tau^{1-\alpha} \vec{v}^{*}, \quad t_{0}<t \leq t_{1}
$$

where $\vec{v}^{*}={ }^{t}\left(v_{1}^{*}, v_{2}^{*}, \ldots, v_{m}^{*}\right), \quad A^{*}=A-\delta(t) E, E$ is a unit matrix. Here we remark that $\operatorname{Re}\left\{i \lambda_{j}(t)-\delta(t)\right\} \geq \delta(t), j=1,2, \ldots, k$, and that $\operatorname{Re}\left\{i \lambda_{j}(t)\right.$ $-\delta(t)\} \leq-\delta(t), j=k+1, \ldots, m$.

We put

$$
S(t)=\sum_{j=1}^{k}\left|v_{j}^{*}(t)\right|^{2}-\sum_{j=k+1}^{m}\left|v_{j}^{*}(t)\right|^{2}
$$

Then we have

$$
\frac{d}{d t} S(t) \geq\left\{2 \delta(t)-C M(t) \tau^{-\frac{1}{m}}\right\} \tau \sum_{j=1}^{m}\left|v_{j}^{*}(t)\right|^{2} .
$$

Define $t_{\tau}$ by

$$
t_{\tau}=\inf \left\{t ; \delta(s)-C M(s) \tau^{-\frac{1}{m}} \geq 0 \text { for any } s \in\left[t, t_{1}\right]\right\}
$$

Then

$$
\frac{d}{d t} S(t) \geq \delta(t) \tau \sum_{j=1}^{m}\left|v_{j}^{*}(t)\right|^{2} \geq \delta(t) \tau S(t), \quad t_{\tau} \leq t \leq t_{1} .
$$

Integrating this inequality, we get

$$
S\left(t_{1}\right) \exp \left\{-\tau \int_{t_{\tau}}^{t_{1}} \delta(s) \mathrm{d} s\right\} \geq S\left(t_{\tau}\right)
$$

Here we remark that

$$
t_{\tau} \longrightarrow t_{0} \quad \text { as } \tau \longrightarrow \infty
$$

Now we define a sequence of solutions of $(2.1)$. Let $v(t ; \tau)$ be the solution of (2.1) which satisfies

$$
v_{1}^{*}\left(t_{\tau} ; \tau\right)=1, \quad v_{j}^{*}\left(t_{\tau} ; \tau\right)=0, \quad j=2,3, \ldots, m .
$$

By (3.10) and (3.11) we have

$$
S\left(t_{1} ; \tau\right) \geq e^{c_{1} \tau}, \quad c_{1} \text { be a positive constant. }
$$


Here $S(t ; \tau)$ stands for $S(t)$ corresponding to $v(t ; \tau)$. Because $S\left(t_{1} ; \tau\right)$ $\leq E\left(t_{1} ; \tau\right)$, we get

$$
E\left(t_{1} ; \tau\right) \geq e^{c_{1} \tau}
$$

At last we consider the equation $(2.1)$ in $\left[t_{0}, t_{\tau}\right]$. It is easy to obtain the estimate

$$
E\left(t_{0} ; \tau\right) \leq E\left(t_{\tau} ; \tau\right) \exp \left\{C_{1}\left(t_{\tau}-t_{0}\right) \tau\right\}
$$

Therefore by (2.13) we get

$$
E(0 ; \tau) \leq E\left(t_{\tau} ; \tau\right) \exp \left\{M_{1} \tau^{1-\alpha}+C_{1}\left(t_{\tau}-t_{0}\right) \tau\right\} .
$$

If we remark that $M\left(t_{\tau}\right) \leq C \tau^{\frac{1}{m}}$, we have

$$
E\left(t_{\tau} ; \tau\right) \leq C_{2} \exp \left\{C_{3}\left(t_{\tau}-t_{0}\right) \tau\right\}
$$

Therefore

$$
E(0 ; \tau) \leq C \exp \left\{M_{1} \tau^{1-\alpha}+M_{2}\left(t_{\tau}-t_{0}\right) \tau\right\}
$$

Since $t_{\tau} \rightarrow t_{0}$ as $\tau \rightarrow \infty$ and $\alpha>0$, (3.14) and (3.15) can not be compatible with the estimate (0.6) whatever the constants $C$ and $p$ we may take.

Thus we have completed the proof of the theorem.

\section{Department of Applied Mathematics, EHIME UNIVERSITY, EHIME}

\section{References}

[1] Lax P. D.: Asymptotic solutions of oscillatory initial value problems, Duke Math. J., 24, 627-646 (1957).

[2] Mizohata S.: Some remarks on the Cauchy problem, J. Math. Kyoto Univ. 1-1, 109-127 (1961).

[3] Mizohata S: The theory of partial differential equations, Iwanami, Tokyo (1965). Cambridge Univ. Press (1973).

[4] Petrowski I. G.: Uber das Cauchysche Problem fur ein System linearer partieller Differentialgleichungen im Gebiete der nichtanalytischen Functionen, Bull. Univ. Etat, Moskow, 1-74 (1938). 